\title{
Spermatocyte Spreading during Meiotic Cell Preparation is a Two Step Process
}

\author{
Razan Muhtadi ${ }^{1}$, Emad A. Ahmed ${ }^{1,2}$ and Harry Scherthan ${ }^{1 *}$ \\ ${ }^{1}$ Inst. für Radiobiologie der Bundeswehr in Verb. mit der Univ. Ulm, Neuherbergstr. 11, D-80937 Munich, Germany \\ ${ }^{2}$ Laboratory of Immunology and Molecular Physiology, Zoology Department, Faculty of Science, Assiut University, Assiut, Egypt
}

* Corresponding author: Harry Scherthan, Inst. für Radiobiologie der Bundeswehr in Verb. mit der Univ. Ulm, Neuherbergstr. 11, D-80937 Munich, Germany, Tel: +49 89 992692 2272; Fax: +49 89992692 2255; E-mail: scherth@web.de

Received date: Mar 20, 2017; Accepted date: Apr 14, 2017; Published date: Apr 26, 2017

Copyright: (c) 2017 Muhtadi, et al. This is an open-access article distributed under the terms of the Creative Commons Attribution License, which permits unrestricted use, distribution, and reproduction in any medium, provided the original author and source are credited.

Keywords: Lipsol; Meiosis; Mouse; Spermatocyte spreading; Testis;

Video microscopy

\begin{abstract}
Cytological techniques have been instrumental for the investigation of meiosis and gametogenesis. Especially high resolution chromatin spreads of male and female germline cells provide for detailed insights into the molecular mechanisms acting during germ cell differentiation. Most spreading techniques for germ cells are done with a hypotonic detergent solution followed by an extended drying period. Using video microscopy we monitored the course of mouse spermatocyte spreading after exposure to graded concentrations of the ionic detergent mixture Lipsol that is known to efficiently spread meiocytes. Our analysis disclosed that spreading of meiotic cells is optimal at a final detergent concentration of about $0.7 \%$ and occurs in two phases. First, cells undergo slow swelling in the dilute detergent solution (lasting up to $>1 \mathrm{~h}$ ) followed by rapid dispersion of the nuclear chromatin over the glass surface just prior to the final evaporation of the water in the solution. These results provide a better understanding of an important technique in meiosis research and identify factors determining the spreading process.
\end{abstract}

\section{Introduction}

Techniques for preparation of meiotic cells are designed to reveal meiotic chromatin and chromosome axes, i.e., lateral/axial elements and the synaptonemal complexes (SCs), in a 2D representation spreadout on a glass surface. This is generally achieved by spreading cells on a hypotonic solution or distilled water/air interface and drying them down on or transferring them to glass slides or EM grids [1,2]. However, there are technical drawbacks associated with the surfacespreading technique on a water interface, e.g., low yield of meiotic nuclei of the less abundant early meiotic prophase stages. Later, techniques achieved spreading by drying-down detergent-containing cell suspensions, which resulted in a higher abundance of meiotic prophase and other cells using gonads or meiotic cells of a variety of species [3-8].

The spreading-out of meiotic cells, from e.g., mammalian gonads, on a glass surface is achieved by preparing and mixing a gonad cell suspension in isotonic buffer with a hypotonic detergent solution containing formaldehyde as the fixative [6]. It has been noted that spreading solutions containing a volume percent of the floor cleaning detergent Lipsol perform well in organisms of different groups $[4,9,10]$. Surface spreading eventually leads to the tight adhesion of the flattened-out nuclear chromatin to the surface of glass slides or EM grids. Spread nuclei thus embody a 2D representation of the meiotic nuclear scaffold and attached chromatin, what in the living cell was a three-dimensional meshwork. The glass-attached chromatin of meiotic spreads has proven highly accessible for immunostaining and/or FISH using wide-field and electron microscopy [6,7,11-14] or atomic force microscopy [15]. It has been realized though, that soluble cellular components like proteins, RNAs, etc. will be lost during the nuclear spreading process, while chromatin-bound molecules will be retained in the spread chromatin.

Usually, the preparation of meiotic spreads requires hours and a limited range of detergent/fixative mixtures have been found to produce the required preparation quality $[5,6,16]$. Here, we were interested in the determinants of the spreading procedure and the dynamics of the transformation from a spherical nucleus to the flat, nearly two-dimensional pancake-like spread chromatin area obtained on the glass slide. To address these questions, we used high resolution video microscopy to study and visualize the dynamics of nuclear transformation during spreading process of mouse spermatocytes and determined factors that are crucial for the spreading process.

\section{Materials and Methods}

\section{Testicular specimens}

Mouse spermatocytes were obtained from wild-type C57BL/6 mice that were kept in compliance with local animal welfare laws, guidelines and policies. Animals were anesthetized and sacrificed by cervical dislocation. Testes were immediately resected and shock-frozen for 5 min in 2-methyl-butane (Sigma) at $-70^{\circ} \mathrm{C}$ and stored at $-80^{\circ} \mathrm{C}$ in the presence of 2-methyl-butane until further use.

\section{Spreading}

Testicular tissue was minced with scalpels in $1 \mathrm{x}$ phosphate buffered saline (PBS), 0.1\% mammalian protease inhibitor (Sigma) in a cold room. The suspension was passed through a nylon pre-separation filter (40 $\mu \mathrm{m}$; Miltenyi Biotec). One microlitre of this cell suspension was pipetted into $20 \mu \mathrm{L}$ PBS on a glass slide. Cells were brought into focus 
and $40 \mu \mathrm{L}$ of ionic detergent solution (0.1-1\% Lipsol; LIP equipment, UK) was added and recording started at 1 frame per second using our FEI live cell microscope setup. Spreading was monitored from the addition of detergent up to $70 \mathrm{~min}$, depending on the detergent concentration and time required for drying down of the spreading solution, the latter being dependent on the temperature and flow of air in the slide environment (e.g., in a fume hood or lab bench); to speed up drying-down in some experiments we mixed only $1 \mu \mathrm{L}$ suspension and $2 \mu \mathrm{L}$ detergent solution. Some dried-down slides were stained with DAPI (4',6-diamidino-2-phenylindole) and subjected to axial element (SYCP3) staining as described previously [17].

\section{Immunofluorescence staining}

Immunostaining experiments were performed as described previously [17] using an antibody against the synaptonemal complex (SC) protein SYCP3 (ab15093; Abcam, Milton, UK). The antibody was used at $1 / 400$ dilution in PCTG (1x PBS, $1 \%$ casein, $0.05 \%$ Tween 20 , $0.1 \%$ gelatin). Secondary Alexa- 488 -labelled antibodies (goat-anti-rabAlexa-488; 1/250) were from Dianova (Hamburg, Germany).

\section{Microscopic evaluation}

Video imaging was done using an Axiovert epifluorescence microscope (Carl Zeiss) with a 40x plan-neofluar lens and a 14/16-bit black-and-white CCD digital camera (Andor Clara; Andor Technology Ltd, UK) controlled by the LiveAquisition (LA) software (FEI/Thermo Fisher Scientific, Waltham, USA). Phase contrast images were recorded at 0.33 or 1 frame per second. Image analysis of time-lapse movies was done using ImageJ (http://imagej.nih.gov/ij/) by interactively determining the diameter of spreading nuclei in the tiff image series. Immunofluorescently stained cells were analyzed and recorded using the ISIS image analysis system (MetaSystems, Altlussheim, Germany).

\section{Results \& Discussion}

Historically, spermatocyte spreading was done placing cells on the surface of a drop of distilled water and thus called 'surface spreading' in 1970s [1,18]. To improve the yield of cells and to standardize the procedure, spreading was thereafter modified by adding detergents to the spreading solution (e.g., Triton X-100, Lipsol, SDS) followed by drying down of the entire cell solution in the presence of a fixative (usually formaldehyde) $[4,6,10]$.

Since we were interested to study the course of the spreading process in detail, we recorded movies of mouse spermatocytes using graded concentrations of the ionic detergent mixture Lipsol that is efficiently spreading meiocytes of species of different kingdoms $[4,10,13,17,19]$. To obtain videos testis cell suspension and detergent solutions were mixed on a glass slide and the spreading process recorded at 0.33 frames per sec. It was important to adjust the density of the testis cell suspension (usually 1/20) so that cells had enough space for spreading-out their chromatin on the glass surface. Analysis of the resulting movies revealed that the cells first float in the detergent solution. With increasing time water evaporates from the solution and cells start to swell changing their anisotropy (they become darker) and randomly attach to the glass surface. When more water evaporates, cells further increase their diameter to eventually rapidly disperse their nuclear chromatin over the glass surface (Movie 1, available at: http:// www.uni-kl.de/FB-Biologie/AG-Scherthan/Movie_1.avi).

The time required for cell attachment and the initiation of spreading was observed to be dependent on the detergent concentration - reducing the Lipsol concentration led to an exponential increase in the time required to attachment and completion of spreading (Figure 1), for instance spreading was complete after 5 min using a $1 \%$ Lipsol solution ( $0.66 \%$ final Lipsol conc.; $n=3$ experiments), but required 20 min using $0.25 \%$ Lipsol ( $0.16 \%$ final conc.) (Figure 1$)$. However, it should be noted that the time frame until spreading initiation will change depending on the surrounding humidity that also has an influence on the quality of spreads obtained [6].



Figure 1: Influence of detergent concentration on the time until final nuclear spreading in our experiments. There was an exponential increase of time required for completion of spreading with decreasing detergent concentration (final conc. in the cell suspension shown). Error bars: Standard error of 2-3 experiments.

Next we analyzed the dynamics of the final fast nuclear spreading process by determining the growing diameter during the final step when a cell starts to disperse its chromatin over the glass surface (Figure 2A). Detailed analysis of the movies revealed that after the relatively long period in solution the nuclear diameter started to rapidly increase reaching its max. diameter in less than 2 minutes, which was observed for all detergent concentrations tested (Figure 2B). The average time a nucleus required for the doubling of its nuclear diameter during spreading was inversely correlated to the Lipsol concentration, with the doubling time for increase of the nuclear diameter requiring 40 to 110 seconds in average. Spreading was fastest with the highest Lipsol concentration - 40 seconds in average in $0.66 \%$ Lipsol and nearly 2 minutes with $0.066 \%$ Lipsol in the cell suspension (Figure 2B).

The above observations suggest that the evaporation of water from the cell suspension slowly increases the concentration of the detergent and, when eventually the volume of the solution is reduced and the critical concentration of detergent for membrane lysis is reached, the nuclei attach to and will disperse their chromatin across the glass surface. To determine whether an increase of the initial detergent concentration would lead to an overall faster spreading process, we tested this in spermatocytes and lymphoblastoid cells. Adding increasing concentrations (1-10\%) of Lipsol to the cell suspension revealed that the latter rapidly swell and rupture in solution, already after $1 \mathrm{sec}$ at a final Lipsol concentration of $\geq 4.4 \%$ in the suspension. At $2.5 \%$, cells took only $\sim 10$ seconds to rupture. Similar experiments with testes suspensions showed that spermatocytes did spread in less than 30 s in a $5 \%$ detergent solution. 


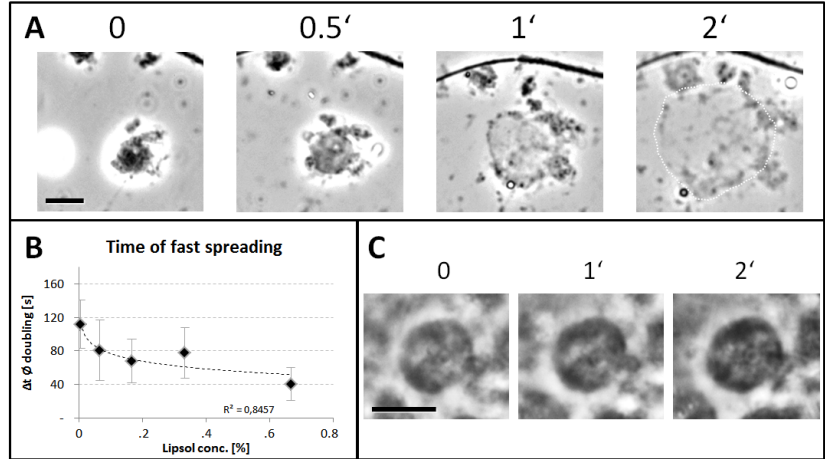

Figure 2: Spreading dynamics. (A) Fast spermatocyte spreading initiates when most of the water has evaporated (0) the cell starts to rapidly increase its diameter, spreading is complete after 2 min (2') in $0.66 \%$ Lipsol solution. The stippled line indicates the outline of the spread chromatin. Bar represents $10 \mu \mathrm{m}$. (B) Influence of the Lipsol concentration on average spermatocyte spreading time (sec; determined from 5-14 nuclei in resp. movies). The fast spreading step takes about 40-110 sec depending on the Lipsol concentration used (final conc. in the cell suspension shown). (C) Impact of formaldehyde in the spreading solution on cell morphology. The spermatocyte nuclear diameter hardly changes over $2 \mathrm{~min}$ in a solution containing $1 \%$ formaldehyde and $0.66 \%$ Lipsol. Bar: $10 \mu \mathrm{m}$.

Peters et al. [6] indicated that the presence of formaldehyde in the spreading solution had beneficial effects on the quality of the spreads. The addition of $1 \%$ formaldehyde to the spreading solution preserved cell morphology and prevented detergent-induced cell rupture in solution (Figure 2C). However, we also observed that fast drying (within approx. one minute) interfered with the chromatin moving across the glass surface, which agrees with the observation of Peters et al. that showed that slow drying down the solution over several hours is beneficial for preparation quality [6].

Since the spreading solutions usually are low in salt $(\sim 46 \mathrm{mM} \mathrm{Na})$, histones and other chromatin components remain attached to the spreading chromatin. The final maximal nucleoid diameters obtained after spreading (up to $70 \mu \mathrm{m}$ ) were usually determined by the density of the cells in suspension, i.e. presence of cells nearby that limited the spreading of the nuclear chromatin (see Movie 1). In some nuclei we could detect thin dark lines within the spreading chromatin, which were reminiscent of synaptonemal complexes (SCs). The latter could be verified by immunostaining for the SC axial element protein SYCP3 (Figure 3). Comparing the extension (diameter) of the DAPI-stained chromatin revealed a similar extension of the of dried-down spread nuclei (average diameter 32.1 $\mu \mathrm{m}+-3.8 \mu \mathrm{m} \quad \mathrm{SD} ; \mathrm{n}=23$ ) of immunofluorescence preparations with formaldehyde added after 5 min and the dynamic video imaging results obtained with $1 \%$ Lipsol solution (av. spermatocyte diameter $31.2+-6.7 ; \mathrm{n}=42$ ).

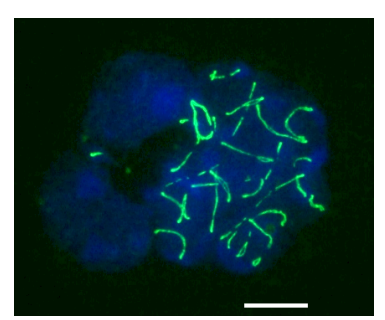

Figure 3: Spermatocyte nucleus and two other nuclei (DAPI, blue) after spreading with $0.66 \%$ Lipsol and IF with SYCP3 antibodies showing SCs and axial cores (green). Bar: $10 \mu \mathrm{m}$.

It has been reported that the sharpness of the SCs and the contrast between the SCs and adherent chromatin varies with changes in humidity and time of drying [6]. In line with this suggestion we noted that the concentration of spreading detergent is a determinant of target unmasking and thus good immuno-stainability, expressed by a weak specific signal and high background in preparations obtained with low $(0.066 \%)$ Lipsol concentrations: Higher concentrations of detergent led to more intense fluorescence signals (Figure 3), suggesting that the release of more soluble chromatin components allow better access of the immunostaining reagents to the remaining proteins in the spreadout chromatin.

In all, our observations disclose that optimal spreading of meiotic cells is best using a low detergent concentration of about $0.66 \%$ and an appropriate cell density. Spreading is a two phase process that first results in slow swelling in solution, followed by a second phase of fast dispersion of the chromatin over the glass surface. The latter occurring when most of the water in the solution has evaporated. Addition of formaldehyde fixative to the spreading solution will influence the chromatin contraction onto the SCs and widths of the obtained nucleoid dimeter ([6], own unpublished observations). Therefore, it is recommended to first add a detergent solution (containing 1\% Lipsol) to the cell suspension and adding fixative solution ( $1 \%$ formaldehyde) after 5 minutes, followed by 30 minute incubation at $4^{\circ} \mathrm{C}$ in a closed box and slow drying down under a fume hood with the lid open.

\section{Acknowledgement}

We thank S. Dürl and C. Adelfalk for technical assistance during early parts of this project and J. Loidl, Univ. of Vienna, Austria, for critical comments on the manuscript. This research was in part supported by a DFG grant to HS (SCHE 350/12-2).

\section{References}

1. Counce SJ, Meyer GF (1973) Differentiation of the synaptonemal complex and the kinetochore in Locusta spermatocytes studied by whole mount electron microscopy. Chromosoma 44: 231-253.

2. Comings DE, Okada TA (1970) Whole Mount Electron Microscopy of Meiotic Chromosomes and the Synaptonemal Complex. Chromosoma 30: 269-286.

3. Speed RM (1982) Meiosis in the foetal mouse ovary. I. An analysis at the light microscope level using surface-spreading. Chromosoma 85: 427-437.

4. Albini SM, Jones GH (1984) Synaptonemal complex-associated centromeres and recombination nodules in plant meiocytes prepared by an improved surface-spreading technique. Exp Cell Res 155: 588-592. 
Citation: Muhtadi R, Ahmed EA, Scherthan H (2017) Spermatocyte Spreading during Meiotic Cell Preparation is a Two Step Process . J Cytol Histol 8: 1000450. doi:10.4172/2157-7099.1000450

Page 4 of 4

5. Bahler J, Wyler T, Loidl J, Kohli J (1993) Unusual nuclear structures in meiotic prophase of fission yeast: a cytological analysis. J Cell Biol 121: 241-256.

6. Peters AH, Plug AW, van Vugt MJ, de Boer P (1997) A drying-down technique for the spreading of mammalian meiocytes from the male and female germline. Chromosome Res 5: 66-68.

7. Rockmill B (2009) Chromosome spreading and immunofluorescence methods in Saccharomyes cerevisiae. Methods Mol Biol 558: 3-13.

8. Dresser MN, Giroux CN (1988) Meiotic chromosome behavior in spread preparations of yeast. Journal of Cell Biology 106: 567-573.

9. Loidl J, Nairz K, Klein F (1991) Meiotic chromosome synapsis in a haploid yeast. Chromosoma 100: 221-228.

10. Loidl J, Schweizer D (1992) Synaptonemal complexes of Xenopus laevis. J Heredity 83: 307-309.

11. Moens PB, Pearlman RE (1989) Satellite DNA I in chromatin loops of rat pachytene chromosomes and in spermatids. Chromosoma 98: 287-294.

12. Heyting C, Dietrich AJ, Moens PB, Dettmers RJ, Offenberg HH, et al. (1989) Synaptonemal complex proteins. Genome 31: 81-87.

13. Scherthan H, Loidl J, Schuster T, Schweizer D (1992) Meiotic chromosome condensation and pairing in Saccharomyces cerevisiae studied by chromosome painting. Chromosoma 101: 590-595.
14. Dresser M, Pisetsky D, Warren R, McCarty G, Moses M (1987) A new method for the cytological analysis of autoantibody specificities using whole-mount, surface-spread meiotic nuclei. J Immunol Methods 104: 111-121.

15. Hausmann M, Liebe B, Perner B, Jerratsch M, Greulich KO, et al. (2003) Imaging of human meiotic chromosomes by scanning near-field optical microscopy (SNOM). Micron 34: 441-447.

16. Dresser ME, Giroux CN (1988) Meiotic chromosome behavior in spread preparations of yeast. J Cell Biol 106: 567-573.

17. Scherthan H, Jerratsch M, Li B, Smith S, Hulten M, et al. (2000) Mammalian meiotic telomeres: protein composition and redistribution in relation to nuclear pores. Mol Biol Cell 11: 4189-4203.

18. Comings DE, Okada TA (1970) Whole mount electron microscopy of meiotic chromosomes and the synaptonmal complex. Chromosoma 30: 269-286.

19. Liebe B, Alsheimer M, Hoog C, Benavente R, Scherthan H (2004) Telomere attachment, meiotic chromosome condensation, pairing, and bouquet stage duration are modified in spermatocytes lacking axial elements. Mol Biol Cell 15: 827-837. 